\title{
The Lax Representation for an Integrable Class of Relativistic Dynamical Systems
}

\author{
M. Bruschi ${ }^{1}$ and F. Calogero ${ }^{2}$ \\ 1 Dipartimento di Fisica, Università di Roma "La Sapienza", I-00185 Roma, Italy \\ 2 Istituto Nazionale di Fisica Nucleare, Sezione di Roma, and \\ Centro Linceo Interdisciplinare di Scienze Matematiche e loro Applicazioni, Accademia dei \\ Lincei, Roma *
}

\begin{abstract}
We exhibit the Lax pair for the class of relativistic dynamical systems recently introduced by Ruijsenaars and Schneider, whose equations of motion $\operatorname{read} \ddot{q}_{j}=\sum_{k=1, k \neq j}^{n} \dot{q}_{j} \cdot \dot{q}_{k} \cdot v\left(q_{j}-q_{k}\right), j=1,2, \ldots, N$, with $v(x)=\mathscr{P}^{\prime}(x) /[B-\mathscr{P}(x)]$, where $B$ is an arbitrary constant and $\mathscr{P}(x)$ the Weierstrass elliptic function.
\end{abstract}

\section{Introduction}

Recently Ruijsenaars and Schneider [1] have introduced a class of integrable dynamical systems, whose interest is underlined by the possibility to interpret these models as describing the one-dimensional motion of $n$ interacting relativistic particles and by their relation to the multisoliton solutions of certain integrable PDEs such as the sine-Gordon equation. The main purpose of this paper is to exhibit the representation of the equations of motion of these systems,

$$
\ddot{q}_{j}=\sum_{\substack{k=1 \\ k \neq j}}^{n} \dot{q}_{j} \dot{q}_{k} v\left(q_{j}-q_{k}\right), \quad q_{j}=q_{j}(t), \quad j=1,2, \ldots, n,
$$

in the Lax form [2],

$$
\dot{\mathbf{L}}=[\mathbf{L}, \mathbf{M}],
$$

where $\mathbf{L}$ and $\mathbf{M}$ are the $n \times n$ matrices,

$$
\begin{gathered}
L_{j k}=\delta_{j k} \dot{q}_{j}+\left(1-\delta_{j k}\right)\left(\dot{q}_{j} \dot{q}_{k}\right)^{1 / 2} \alpha\left(q_{j}-q_{k}\right), \\
M_{j k}=\delta_{j k} \sum_{\substack{m=1 \\
m \neq j}}^{n} \dot{q}_{m} \beta\left(q_{j}-q_{m}\right)+\left(1-\delta_{j k}\right)\left(\dot{q}_{j} \dot{q}_{k}\right)^{1 / 2} \gamma\left(q_{j}-q_{k}\right) .
\end{gathered}
$$

* For the 3 academic years 1983-1986 
As shown below, the most general function $v(x)$ that allows us to recast (1.1) in the form (1.2) with (1.3) is (up to a trivial rescaling of the dynamical variables $q_{j}$ )

$$
v(x)=-d\{\ln [\mathscr{P}(x)-B]\} / d x=\mathscr{P}^{\prime}(x) /[B-\mathscr{P}(x)],
$$

with $B$ an arbitrary constant and $\mathscr{P}(x)=\mathscr{P}\left(x \mid \omega, \omega^{\prime}\right)$ the Weierstrass function [3]. Special cases of $v(x)$ are

$$
\begin{gathered}
v(x)=2 \operatorname{cotgh}(x) /\left[1-C \sinh ^{2}(x)\right], \\
v(x)=2 /\left[x\left(1-C x^{2}\right)\right],
\end{gathered}
$$

with $C$ an arbitrary constant. The corresponding expressions of the functions $\alpha(x)$, $\beta(x)$, and $\gamma(x)$, see (1.3) and (1.4), are given below.

To obtain these results we have solved the functional equation,

$$
\alpha(x) \alpha^{\prime}(y)-\alpha^{\prime}(x) \alpha(y)=[\alpha(x+y)-\alpha(x) \alpha(y)][\eta(x)-\eta(y)],
$$

where $\alpha(x)$ and $\eta(x)$ are two (a priori unknown) functions to be determined, and of course $\alpha^{\prime}(x)=d \alpha(x) / d x$; this result may present a certain mathematical interest, the more so since it is obtained by solving the related (non-differential) functional equation

$$
\alpha(x+y)=\alpha(x) \alpha(y)+\varphi(x) \varphi(y) \psi(x+y),
$$

where $\alpha(x), \varphi(x)$, and $\psi(x)$ are three (a priori unknown) functions.

Let us finally emphasize that the integrability of the system (1.1) with (1.4a) had already been demonstrated in [1], through the explicit display of $n$ functionally independent constants of the motion (it remains however to be proven that these $n$ constants are in involution). Moreover, in the special cases mentioned above, Ruijsenaars and Schneider [1] had correctly guessed the form of the Lax matrix $\mathbf{L}$, being guided by the requirement that the eigenvalues of $\mathbf{L}$ coincide with their $n$ constants of the motion (or some suitable combinations of them); in these special cases they also proved that the $n$ constants of the motion are in involution. Now that the Lax form (1.2) of the equations of motion is explicitly established, it is of course a trivial consequence of it that the $n$ eigenvalues of $\mathbf{L}$ are constants of the motion. Presumably other results of [1], as well as new results, could also now be obtained in a more straightforward manner using the Lax pair; but in this paper we limit our treatment to the exhibition of its explicit form.

\section{The Lax Pair}

In this paper we discuss the dynamical system characterized by the equations of motion

$$
\ddot{q}_{j}=\sum_{\substack{k=1 \\ k \neq j}}^{n} \dot{q}_{j} \dot{q}_{k} v\left(q_{j}-q_{k}\right), \quad j=1,2, \ldots, n .
$$

Here of course $q_{j}=q_{j}(t)$, and the superimposed dot denotes $t$-differentiation. In particular we ascertain below that this system can be recast in the Lax form (1.2) if

$$
v(x)=-d\{\ln [B-\mathscr{P}(x)]\} / d x=\mathscr{P}^{\prime}(x) /[B-\mathscr{P}(x)],
$$


where $B$ is an arbitrary constant and $\mathscr{P}(x)$ is the Weierstrass function [3],

$$
\mathscr{P}(x) \equiv \mathscr{P}\left(x \mid \omega, \omega^{\prime}\right)=x^{-2}+\sum_{m, n=-\infty}^{+\infty}\left[\left(x+2 m \omega+2 n \omega^{\prime}\right)^{-2}-\left(2 m \omega+2 n \omega^{\prime}\right)^{-2}\right] .
$$

The prime appended to the summation symbol is of course a reminder of the prescription to omit from the sum the (singular) term with $m=n=0$. [1],

It is plain to verify that the equations of motion (2.1) coincide with Eq. (3.12) of

$$
\begin{gathered}
\ddot{q}_{j}=\sum_{\substack{k=1 \\
k \neq j}}^{n} F_{j k}, \\
F_{j k}=2 \dot{q}_{j} \dot{q}_{k}\left(V_{j}\right)^{-1} V_{j, k}, \\
V_{j}(q)=\prod_{\substack{k=1 \\
k \neq j}}^{n} f\left(q_{j}-q_{k}\right), \\
V_{j, k} \equiv \partial V_{j}(q) / \partial q_{k},
\end{gathered}
$$

via the identification

$$
v(x)=-2 d \ln f(x) / d x=-2 f^{\prime}(x) / f(x) .
$$

It can be easily verified that the equations of motion (2.1) are equivalent to the matrix Lax equation (1.2), provided the two $n \times n$ matrices $\mathbf{L}$ and $\mathbf{M}$ are defined by (1.3) and (1.4), namely

$$
\begin{gathered}
L_{j k}=\dot{q}_{j} \text { if } j=k, \\
L_{j k}=\left(\dot{q}_{j} \dot{q}_{k}\right)^{1 / 2} \alpha\left(q_{j}-q_{k}\right) \text { if } j \neq k, \\
M_{j k}=\sum_{\substack{m=1 \\
m \neq j}}^{n} \dot{q}_{m} \beta\left(q_{j}-q_{m}\right) \text { if } j=k, \\
M_{j k}=\left(\dot{q}_{j} \dot{q}_{k}\right)^{1 / 2} \gamma\left(q_{j}-q_{k}\right) \text { if } j \neq k,
\end{gathered}
$$

with

$$
v(x)=d \ln [A(x)-1] / d x=A^{\prime}(x) /[A(x)-1],
$$

where

$$
A(x) \equiv \alpha(x) \alpha(-x),
$$

and with the three functions $\alpha(x), \beta(x)$, and $\gamma(x)$ satisfying the following restrictions:

$$
\begin{gathered}
\beta(x)=\beta(-x), \\
\gamma(x)=\alpha^{\prime}(x)-\alpha(x) \eta(x), \\
\alpha(x) \alpha^{\prime}(y)-\alpha^{\prime}(x) \alpha(y)=[\alpha(x+y)-\alpha(x) \alpha(y)][\eta(x)-\eta(y)],
\end{gathered}
$$

with

$$
\eta(x)=\beta(x)+\frac{1}{2} v(x) .
$$


Note that (2.8) with (2.9) imply

$$
v(x)=-v(-x) .
$$

Clearly the only substantive constraint is provided by the functional equation (2.12): indeed, once two functions $\alpha(x)$ and $\eta(x)$ that satisfy it have been found, it is trivial to obtain $\gamma(x)$, see (2.11), as well as $\beta(x)$, which is merely the even part of $\eta(x)$,

$$
\beta(x)=\frac{1}{2}[\eta(x)+\eta(-x)],
$$

see (2.10), (2.13), and (2.14). As for the consistency of (2.13) with (2.8), it is plain to verify, setting $y=-x$ in (2.12) and using (2.13), (2.10), (2.14), and (2.8), that it is automatically implied by (2.12) and by the constraint

$$
\alpha(0)=1,
$$

that is therefore hereafter assumed to hold. [It is actually easy to show that the assumption that $\alpha(0)$ and $\alpha^{\prime}(0)$ be finite is sufficient to obtain (2.16) from (2.12).]

The functional equation (2.12) admits obviously the trivial solution $\alpha(x)=\exp (\varrho x)$, with $\varrho$ an arbitrary constant and $\eta(x)$ an arbitrary function. This corresponds to the free motion with $v(x)=0$ [see (2.1)]. This trivial case is hereafter ignored.

\section{The Functional Equation}

In this section we discuss and solve the functional equation (2.12) with (2.16).

Let us mention first of all three invariance properties of this functional equation. If $\alpha(x)$ and $\eta(x)$ satisfy (2.12) and (2.16), so do $\tilde{\alpha}(x)$ and $\tilde{\eta}(x)$ defined as follows:

$$
\begin{aligned}
& \tilde{\alpha}(x)=\alpha(\lambda x), \\
& \tilde{\eta}(x)=\lambda \eta(\lambda x), \\
& \tilde{v}(x)=\lambda v(\lambda x) ; \\
& \tilde{\alpha}(x)=\alpha(x) \exp (\varrho x), \\
& \tilde{\eta}(x)=\eta(x), \\
& \tilde{v}(x)=v(x) ; \\
& \tilde{\alpha}(x)=1 / \alpha(x), \\
& \tilde{\eta}(x)=\eta(x)-\alpha^{\prime}(x) / \alpha(x), \\
& \tilde{v}(x)=v(x) / A(x)=v(x) /[\alpha(x) \alpha(-x)] .
\end{aligned}
$$

These properties are easily verified by direct substitution in (2.12) and (2.16); of course more complicated transformations can be obtained combining those given above. Note that in each case we have also indicated how the function $v(x)$, see (2.1) and (2.8), changes.

It is moreover plain that $\eta(x)$ is defined up to an arbitrary additive constant: indeed (2.12) is clearly invariant under the transformation $\eta(x) \rightarrow \tilde{\eta}(x)=\eta(x)+c$. Note that the corresponding transformations, $\beta(x) \rightarrow \widetilde{\beta}(x)=\beta(x)+c$ (see (2.15)), $\gamma(x)$ 
$\rightarrow \tilde{\gamma}(x)=\gamma(x)-c . \alpha(x)(\operatorname{see}(2.11))$, implying $\mathbf{M} \rightarrow \tilde{\mathbf{M}}=\mathbf{M}+c p \mathbf{I}-c \mathbf{L}$ (see (2.7); here of course $p \equiv \sum_{j=1}^{n} \dot{q}_{j}$ and $\mathbf{I}$ is the unit matrix), do not spoil the validity of the Lax equation (1.2).

Next, let us obtain from (2.12) and (2.16) an expression of $\eta(x)$ in terms of $\alpha(x)$ and its derivatives, and, most importantly, a differential equation that $\alpha(x)$ must satisfy. To this end we consider the behavior of $\alpha(\varepsilon)$ and $\eta(\varepsilon)$ as $\varepsilon \rightarrow 0$, which, as it is easily seen, is given by the following expansions:

$$
\begin{gathered}
\alpha(\varepsilon)=1+\alpha_{1} \varepsilon+\frac{1}{2} \alpha_{2} \varepsilon^{2}+\frac{1}{6} \alpha_{3} \varepsilon^{3}+O\left(\varepsilon^{4}\right), \\
\eta(\varepsilon)=1 / \varepsilon+\eta_{0}+\eta_{1} \varepsilon+O\left(\varepsilon^{2}\right) .
\end{gathered}
$$

Here the 5 coefficients $\alpha_{1}, \alpha_{2}, \alpha_{3}, \eta_{0}$, and $\eta_{1}$ are of course a priori arbitrary.

Set now $y=\varepsilon$ in (2.12), expand in $\varepsilon$ and equate the coefficients of the powers of $\varepsilon$ to zero. The coefficient of $\varepsilon^{0}$ vanish automatically; this merely guarantees the consistency of the ansatz (3.4). The coefficient of $\varepsilon^{1}$ yields the following expression of $\eta(x)$ in terms of $\alpha(x)$ :

$$
\eta(x)=\eta_{0}+\frac{1}{2}\left[\alpha^{\prime \prime}(x)-2 \alpha_{1} \alpha^{\prime}(x)+\alpha_{2} \alpha(x)\right] /\left[\alpha^{\prime}(x)-\alpha_{1} \alpha(x)\right] .
$$

The constant $\eta_{0}$ is of course arbitrary and plays no role.

Finally, the coefficient of $\varepsilon^{2}$ yields, after using (3.5), the following nonlinear ODE for $\alpha(x)$ :

$$
2\left(\alpha^{\prime}-\alpha_{1} \alpha\right) \alpha^{\prime \prime \prime}-3\left(\alpha^{\prime \prime}-2 \alpha_{1} \alpha^{\prime}\right) \alpha^{\prime \prime}+a\left(\alpha^{\prime}\right)^{2}+b \alpha \alpha^{\prime}+c \alpha^{2}=0,
$$

with

$$
\begin{gathered}
a=6\left(2 \eta_{1}-\alpha_{2}\right), \\
b=4\left(\alpha_{3}-6 \alpha_{1} \eta_{1}\right), \\
c=3 \alpha_{2}^{2}-4 \alpha_{1} \alpha_{3}+12 \alpha_{1}^{2} \eta_{1} .
\end{gathered}
$$

The ODE (3.6) contains the 4 parameters $\alpha_{1}, a, b$, and $c$, whose arbitrariness is tantamount to the a priori arbitrariness of the 4 parameters $\alpha_{1}, \alpha_{2}, \alpha_{3}$, and $\eta_{1}$ [see (3.7)]. And it may be verified that (3.6) is consistent with the fact that the $\alpha_{j}$ 's are just the coefficients of the expansion (3.4a).

Since the ODE (3.6) is a consequence of the functional equation (2.12) [and of the condition (2.16); see (3.4a)], it is plain that the most general solution of this ODE (with the condition (2.16)) includes all solutions of (2.12) and (2.16). Such a solution must contain 6 a priori arbitrary constants, since (3.6) features 4 a priori arbitrary constants and is a third order ODE, and (2.16) imposes one condition $(6=4+3-1$; of course the 6 a priori free parameters contained in such a general solution determine the values of the 4 parameters $\alpha_{1}, a, b$, and $c$ in (3.6)).

We have found that such a solution reads as follows:

$$
\alpha(x)=\exp (\varrho x) \frac{\sigma\left(\mu \mid \omega, \omega^{\prime}\right) \sigma\left(\lambda x+v \mid \omega, \omega^{\prime}\right)}{\sigma\left(v \mid \omega, \omega^{\prime}\right) \sigma\left(\lambda x+\mu \mid \omega, \omega^{\prime}\right)} .
$$

Here the 6 parameters $\lambda, \mu, v, \varrho, \omega$, and $\omega^{\prime}$ are arbitrary (except for the trivial requirement $\left.\sigma\left(v \mid \omega, \omega^{\prime}\right) \neq 0\right)$, and $\sigma\left(z \mid \omega, \omega^{\prime}\right)$ is the Weierstrass $\sigma$-function [3]. Let us 
recall that $\sigma\left(z \mid \omega, \omega^{\prime}\right)$ is an entire odd function of $z$, related to the Weierstrass function $\mathscr{P}\left(z \mid \omega, \omega^{\prime}\right)$ by the differential equation

$$
\left[\sigma^{\prime}(z) / \sigma(z)\right]^{\prime}=-\mathscr{P}(z), \quad \sigma(0)=\sigma^{\prime \prime}(0)=0,
$$

and defined by the formula

$$
\sigma(z)=\sum_{m, n=0}^{\infty} a_{m, n}\left(\frac{1}{2} g_{2}\right)^{m}\left(2 g_{3}\right)^{n} z^{1+4 m+6 n} /(1+4 m+6 n) !,
$$

with the "invariants" $g_{2}$ and $g_{3}$ related to the "half-periods" $\omega$ and $\omega$ ' by the formulae

$$
\begin{aligned}
& g_{2}=60 \sum_{m, n=-\infty}^{+\infty}\left(2 m \omega+2 n \omega^{\prime}\right)^{-4}, \\
& g_{3}=140 \sum_{m, n=-\infty}^{+\infty}\left(2 m \omega+2 n \omega^{\prime}\right)^{-6},
\end{aligned}
$$

and the numerical coefficients $a_{m, n}$ determined by the recursion relations $a_{m, n}=3(m+1) a_{m+1, n-1}+\frac{16}{3}(n+1) a_{m-2, n+1}-\frac{1}{3}(2 m+3 n-1)(4 m+6 n-1) a_{m-1, n}$,

with $a_{0,0}=1$ and the convention that $a_{m, n}$ vanish if either subscript is negative.

Note that the expression (3.8) is clearly invariant under the 3 transformations (3.1), (3.2), and (3.3), whose only effect is to cause a redefinition of some parameters.

The verification that (3.8) satisfies (3.6) is a cumbersome task that is left as an exercise for the diligent reader. This result is in fact not required for our purposes, since it is actually the functional equation (2.12) that we must solve, and we know of course that any function $\alpha(x)$ that satisfies (2.12) and (2.16) must automatically satisfy (3.6). Thus the expression (3.8) of $\alpha(x)$ may be considered to be just a convenient ansatz for solving the functional equation (2.12) [note that (3.8) satisfies automatically (2.16)]; an ansatz that is certainly sufficient to capture the most general solution of (2.12), since it contains 6 free parameters, which is the maximal number consistent with (3.6) and (2.16), hence with (2.12) and (2.16).

As it happens, the expression (3.8) of $\alpha(x)$, together with an appropriate choice of $\eta(x)$ (see below), does satisfy the functional equation (2.12), with no (nontrivial) restriction on the 6 parameters $\lambda, \mu, v, \varrho, \omega$, and $\omega^{\prime}$. To prove this result it is actually sufficient, in view of the properties (3.1) and (3.2), to consider the subclass of solutions

$$
\alpha(x)=\frac{\sigma(\mu) \cdot \sigma(x+v)}{\sigma(v) \cdot \sigma(x+\mu)},
$$

which obtains from (3.8) setting $\varrho=0$ and $\lambda=1$. Note that this expression contains 4 free parameters, $\mu, v, \omega$, and $\omega^{\prime}$; the dependence on the last two has not been explicitly displayed (we will often take advantage of this notational simplification).

The expression of $\eta(x)$ that, together with (3.13), satisfies (2.12), reads as follows (up, of course, to an arbitrary additive constant):

$$
\eta(x)=-\frac{1}{2} \frac{\mathscr{P}^{\prime}(x)-\mathscr{P}^{\prime}(\mu)}{\mathscr{P}(x)-\mathscr{P}(\mu)} .
$$


The explicit verification that (3.13) and (3.14) satisfy (2.12) is another cumbersome task that we leave to the diligent reader. Our preferred route to prove this result is via another functional equation, that contains no derivatives and is equivalent to (2.12). This new functional equation reads as follows:

$$
\alpha(x+y)=\alpha(x) \alpha(y)+\varphi(x) \varphi(y) \psi(x+y) .
$$

It is easy to prove (see Appendix) that this functional equation, with $\psi(z)$ a priori arbitrary and $\varphi(x)$ related to $\eta(x)$ as follows,

$$
\varphi(x)=\exp \left[\int^{x} d x^{\prime} \eta\left(x^{\prime}\right)\right]
$$

is equivalent to (2.12).

In the investigation of the functional equation (3.15) the 3 functions $\alpha(z), \varphi(z)$, and $\psi(z)$ should be viewed as a priori arbitrary. This implies the possibility to rewrite $(3.15)$ in many alternative forms, for instance

$$
\alpha(x) \alpha(y) / \alpha(x+y)=1+\varphi(x) \varphi(y) / \chi(x+y),
$$

which corresponds to (3.15) via the identification

$$
\chi(x)=-\alpha(x) / \psi(x) .
$$

It is moreover plain that, if $\alpha(x), \varphi(x)$, and $\psi(x)$ satisfy (3.15), so do

$$
\begin{gathered}
\tilde{\alpha}(x)=\alpha(\lambda x) \exp (\varrho x), \\
\tilde{\varphi}(x)=a \varphi(\lambda x) \exp \left[\left(\varrho+\varrho^{\prime}\right) x\right], \\
\tilde{\psi}(x)=a^{-2} \psi(\lambda x) \exp \left(-2 \varrho^{\prime} x\right),
\end{gathered}
$$

with $a, \varrho$, and $\varrho^{\prime}$ arbitrary constants. This invariance property corresponds clearly to those displayed by (3.1) and (3.2); note incidentally that the possibility to rescale $\varphi$, namely to choose arbitrarily the constant a in $(3.19 \mathrm{~b})$, corresponds to the possibility to add an arbitrary constant to $\eta(x)$ and to choose arbitrarily the lower limit of integration in the r.h.s. of (3.16). As for the transformation equivalent to (3.3), it reads

$$
\begin{gathered}
\tilde{\alpha}(x)=1 / \alpha(x), \\
\tilde{\varphi}(x)=\varphi(x) / \alpha(x), \\
\tilde{\psi}(x)=-\psi(x) / \alpha(x) .
\end{gathered}
$$

It is now easy to verify (see Appendix) that the expression (3.13) of $\alpha(x)$ satisfies (3.15), with

$$
\begin{gathered}
\varphi(x)=\sigma(\mu) \cdot \sigma(x) /[\sigma(v) \cdot \sigma(x+\mu)], \\
\psi(z)=\sigma(v-\mu) \cdot \sigma(z+\mu+v) /[\sigma(\mu) \cdot \sigma(z+\mu)] .
\end{gathered}
$$

\section{Explicit Expressions and Special Cases}

The results given above imply that the most general integrable dynamical system of type (2.1), that may be cast in the Lax form (1.2) with the Lax pair given by the 
ansatz (2.6), (2.7), is characterized, up to a trivial rescaling of the dynamical variables $q_{j}$, by the function $v(x)$ of Eq. (2.2), with the identification

$$
B=\mathscr{P}(\mu) \text {. }
$$

This is implied, via (2.8), by the explicit expressions

$$
\begin{gathered}
A(x)=\frac{\mathscr{P}(x)-\mathscr{P}(v)}{\mathscr{P}(x)-\mathscr{P}(\mu)}, \\
A(x)-1=\frac{\mathscr{P}(\mu)-\mathscr{P}(v)}{\mathscr{P}(x)-\mathscr{P}(\mu)},
\end{gathered}
$$

that follow readily, via (A.6), from the expression (3.13) of $\alpha(x)$. Note that $v(x)$ does not depend on the quantity $v$; it depends only on the 3 parameters $\omega, \omega^{\prime}$, and $\mu$ (or $B$; see (4.1)).

It is a general feature of the function $v(x)$, see (2.2), to have a pole at the origin, with residue 2 :

$$
\lim _{x \rightarrow 0}[x v(x)]=2
$$

The corresponding Lax pair is given by (2.6) and (2.7) with

$$
\begin{gathered}
\alpha(x)=\exp (\varrho x) \cdot \sigma(\mu) \cdot \sigma(x+v) /[\sigma(v) \cdot \sigma(x+\mu)], \\
\beta(x)=c+\frac{1}{2} \mathscr{P}^{\prime}(\mu) /[\mathscr{P}(x)-\mathscr{P}(\mu)], \\
\gamma(x)=\alpha(x) \cdot[\zeta(x+v)-\zeta(x)] .
\end{gathered}
$$

The function $\zeta$ in the last formula is the Weierstrass $\zeta$-function, characterized by the two formulae

$$
\begin{gathered}
\zeta^{\prime}(z)=-\mathscr{P}(z), \\
\lim _{z \rightarrow 0}\left[\zeta(z)-z^{-1}\right]=0 .
\end{gathered}
$$

The Lax pair given by these formulae, and by (2.6) and (2.7), contains the 6 parameters $\varrho, \mu, v, c, \omega$, and $\omega^{\prime}$; but, as noted above, only 3 of these, $\mu, \omega$, and $\omega^{\prime}$, affect the corresponding $v(x)$. Note that these formulae correspond to the choice $\lambda=1$ in (3.8); a different value of $\lambda$ amounts merely to rescaling the dynamical variables $q_{j}$ [see (2.1) and (3.1c)].

Let us also exhibit the formulae for two special cases:

$$
\begin{gathered}
\omega=-i \omega^{\prime}=\infty, \quad \varrho=0, \quad c=-i r, \quad \mu^{-1}=i r, \quad v^{-1}=i s \\
\sigma(x)=x, \quad \zeta(x)=x^{-1}, \quad \mathscr{P}(x)=x^{-2} \\
\alpha(x)=(1+i s x) /(1+i r x), \quad \beta(x)=-i r /\left(1+r^{2} x^{2}\right), \quad \gamma(x)=-1 /[x(1+i r x)] \\
v(x)=2 /\left[x\left(1+r^{2} x^{2}\right)\right] \\
\eta(x)=-\zeta(\mu)-\frac{1}{2} \frac{\mathscr{P}^{\prime}(x)-\mathscr{P}^{\prime}(\mu)}{\mathscr{P}(x)-\mathscr{P}(\mu)}=\frac{1}{x(1+i r x)}
\end{gathered}
$$




$$
\begin{gathered}
\varphi(x)=\frac{\mu x}{v(x+\mu)}, \\
\psi(x)=\frac{(v-\mu)(x+\mu+v)}{\mu(x+\mu)} ; \\
\omega=\infty, \quad \omega^{\prime}=\frac{i \pi}{2}, \quad \varrho=0, \quad c=-\operatorname{cotgh}(\mu),
\end{gathered}
$$

$$
\sigma(x)=\sinh (x) \exp \left(-\frac{x^{2}}{6}\right), \quad \zeta(x)=-\frac{1}{3} x+\operatorname{cotgh}(x), \quad \mathscr{P}(x)=\frac{1}{3}+[\sinh (x)]^{-2},
$$$$
\alpha(x)=\exp [(\mu-v) x / 3] \sinh (\mu) \sinh (x+v) /[\sinh (v) \sinh (x+\mu)],
$$$$
\beta(x)=\frac{1}{2} \sinh (2 \mu) /\left[\sinh ^{2}(x)-\sinh ^{2}(\mu)\right],
$$$$
\gamma(x)=-\alpha(x)\{v / 3+\sinh (v) /[\sinh (x) \sinh (x+v)]\},
$$$$
v(x)=2 \sinh ^{2}(\mu) \operatorname{cotgh}(x) /\left[\sinh ^{2}(\mu)-\sinh ^{2}(x)\right],
$$$$
\eta(x)=-\zeta(\mu)-\frac{1}{2} \frac{\mathscr{P}^{\prime}(x)-\mathscr{P}^{\prime}(\mu)}{\mathscr{P}(x)-\mathscr{P}(\mu)}=\frac{\sinh (\mu)}{\sinh (x) \sinh (x+\mu)},
$$$$
\varphi(x)=\exp (\mu x / 3) \sinh (\mu) \sinh (x) /[\sinh (v) \sinh (x+\mu)],
$$$$
\psi(x)=\exp (-v x / 3) \sinh (v-\mu) \sinh (x+\mu+v) /[\sinh (\mu) \sinh (x+\mu)] .
$$

Clearly (4.6d) and (4.7d) coincide, up to a notational change, with (1.4c) and (1.4b).

Finally, let us return to the general case, to remark that, taking advantage of the well known relations of the Weierstrass functions with the Jacobian elliptic functions, of these among themselves, and of the freedom implied by the transformation (3.2), it is possible to introduce several alternative definitions of the function $\alpha(x)$, all of which correspond to the same function $v(x)$. For instance the following 3 expressions of $\alpha(x)$ yield [via (2.8) and (2.9)] the same function $v(x)$ [see (2.2) and (4.1)],

$$
\begin{gathered}
v(x)=\mathscr{P}^{\prime}(x) /[\mathscr{P}(\mu)-\mathscr{P}(x)] ; \\
\alpha(x)=\operatorname{sn}(\mu) / \operatorname{sn}(x+\mu), \\
\alpha(x)=\operatorname{sn}(\mu) \cdot \operatorname{cn}(x+\mu) /[\operatorname{cn}(\mu) \cdot \operatorname{sn}(x+\mu)], \\
\alpha(x)=\operatorname{sn}(\mu) \cdot \operatorname{dn}(x+\mu) /[\operatorname{dn}(\mu) \cdot \operatorname{sn}(x+\mu)] ;
\end{gathered}
$$

while the following 3 expressions of $\alpha(x)$,

$$
\begin{gathered}
\alpha(x)=c n\left(x+\mu-\omega^{\prime}\right) / c n\left(\mu-\omega^{\prime}\right), \\
\alpha(x)=d n\left(x+\mu-\omega^{\prime}\right) / d n\left(\mu-\omega^{\prime}\right), \\
\alpha(x)=\frac{d n\left(\mu+\omega+\omega^{\prime}\right) c n\left(x+\mu+\omega+\omega^{\prime}\right)}{c n\left(\mu+\omega+\omega^{\prime}\right) d n\left(x+\mu+\omega+\omega^{\prime}\right)},
\end{gathered}
$$

yield merely a rescaled version of (4.8), namely

$$
v(x)=-\mathscr{P}^{\prime}(\lambda x) /[\mathscr{P}(\lambda x)-\mathscr{P}(\lambda \mu)],
$$


with

$$
\lambda=\left[\mathscr{P}(\omega)-\mathscr{P}\left(\omega^{\prime}\right)\right]^{-1 / 2} .
$$

Of course in these formulae, as above, $\omega$ and $\omega^{\prime}$ are the two half periods of the Weierstrass $\mathscr{P}$-function and of the 3 Jacobian elliptic functions $s n, c n$, and $d n$, while $\mu$ is an arbitrary parameter. The calculation of the corresponding expressions for $\eta(x)$ [see (2-12) or (3.5)], $\beta(x)$ and $\gamma(x)$ [see (2.7), (2.15), and (2.11)], is left as an exercise for the diligent reader.

\section{Envoi}

As already mentioned in the Introduction, it is possible (indeed likely) that the availability of the Lax pair make it now possible to reobtain in a more straightforward manner some of the results of [1]; for instance it can be used to solve the equations of motion (2.1). Indeed, using these results, it has already been possible to extend some of these models to include "external forces" [4]; a result which is also announced in a Note added to [1].

An interesting open problem has to do with the relations of the results described here with previous results obtained by one of us $[5,6]$. In particular the very special case (4.6) with $r=0$ (or, equivalently, $(1.4 \mathrm{c})$ with $C=0$ ) features the equations of motion

$$
\ddot{q}_{j}=2 \sum_{\substack{k=1 \\ k \neq j}}^{n} \dot{q}_{j} \dot{q}_{k} /\left(q_{j}-q_{k}\right), \quad j=1,2, \ldots, n,
$$

and these are a special case (but in quite another sense) of the equations of motion that have been shown to be integrable (and have actually been integrated) in [5]. The connection between these two classes of integrable dynamical systems, of which (5.1) (as well as the equations of motion corresponding to (1.4b) with $C=0$ ) are special cases, is therefore an interesting open field of investigation, whose first goal should be to clarify the relation among the results and the approaches of [1], of this paper, of $[5,6]$ and of its recent generalizations [7-9].

\section{Appendix}

The first task is to prove the equivalence of (2.12) and (3.15). To this end set

$$
F(x, y)=\ln [1-\alpha(x) \alpha(y) / \alpha(x+y)],
$$

and note that (2.12) then implies

$$
F_{x}(x, y)-F_{y}(x, y)=\eta(x)-\eta(y),
$$

where the subscripts denote partial differentiation. This first order linear PDE for $F$ has the general solution

$$
F(x, y)=H(x)+H(y)+g(x+y)
$$

with $g(z)$ an arbitrary function and

$$
H(x)=\int^{x} d\left(x^{\prime}\right) \eta\left(x^{\prime}\right)
$$


But (A.1) with (A.3) and (A.4) imply (3.15) with (3.16) and

$$
\psi(z)=\alpha(z) \exp [g(z)] \text {. Q.E.D. }
$$

The second task is to prove that (3.13) satisfies (3.15), with (3.21) and (3.22) (note that these two expressions are now derived, rather than assumed). The main tool to obtain this result is the formula [3]

$$
\sigma\left(z_{1}+z_{2}\right) \cdot \sigma\left(z_{1}-z_{2}\right)=\sigma^{2}\left(z_{1}\right) \cdot \sigma^{2}\left(z_{2}\right) \cdot\left[\mathscr{P}\left(z_{2}\right)-\mathscr{P}\left(z_{1}\right)\right] .
$$

Set

$$
\alpha(x+y) / \alpha(x) \alpha(y)-1=N / D
$$

Then, using (3.13),

$$
\begin{gathered}
N=\sigma(v) \cdot \sigma(x+\mu) \cdot \sigma(y+\mu) \cdot \sigma(x+y+v)-(\mu \leftrightarrow v), \\
D=\sigma(\mu) \cdot \sigma(x+v) \cdot \sigma(y+v) \cdot \sigma(x+y+\mu) .
\end{gathered}
$$

Now note that (A.6) implies

$$
\begin{gathered}
\sigma(\mu) \sigma(x+y+\mu)=\sigma^{2}\left[\mu+\frac{(x+y)}{2}\right] \cdot \sigma^{2}\left[\frac{1}{2}(x+y)\right]\left\{\mathscr{P}_{[(x+y) / 2]}-\mathscr{P}_{[\mu+(x+y) / 2]}\right\} \\
\sigma(x+\mu) \cdot \sigma(y+\mu)=\sigma^{2}\left[\mu+\frac{1}{2}(x+y)\right] \cdot \sigma^{2}\left[\frac{1}{2}(x+y)\right] \cdot\left\{\mathscr{P}_{[(x+y) / 2]}-\mathscr{P}_{[\mu+(x+y) / 2]}\right\},
\end{gathered}
$$

and of course analogous formulae with $\mu$ replaced by $v$. From these and (A.8) there obtains

$$
N=\left\{\sigma\left[\mu+\frac{1}{2}(x+y)\right] \cdot \sigma\left[v+\frac{1}{2}(x+y)\right] \cdot \sigma\left[\frac{1}{2}(x-y)\right] \cdot \sigma\left[\frac{1}{2}(x+y)\right]\right\}^{2} \cdot N_{1},
$$

with

$$
\begin{aligned}
N_{1} & =\left\{\mathscr{P}_{[(x+y) / 2]}-\mathscr{P}_{[v+(x+y) / 2]}\right\}\left\{\mathscr{P}_{[(x-y) / 2]}-\mathscr{P}_{[\mu+(x+y) / 2}\right\}-(\mu \leftrightarrow v) \\
& =\left\{\mathscr{P}_{[(x+y) / 2]}-\mathscr{P}_{[(x-y) / 2]}\right\}\left\{\mathscr{P}_{[v+(x+y) / 2]}-\mathscr{P}_{[\mu+(x+y) / 2]}\right\} \\
& =\frac{\sigma(x) \cdot \sigma(y) \cdot \sigma(v-\mu) \cdot \sigma(x+y+\mu+v)}{\left\{\sigma\left[\frac{1}{2}(x+y)\right] \cdot \sigma\left[\frac{1}{2}(x-y)\right] \cdot \sigma\left[\mu+\frac{1}{2}(x+y)\right] \sigma\left[v+\frac{1}{2}(x+y)\right]\right\}^{2}} .
\end{aligned}
$$

To perform the last step we have again used (A.6).

The last two equations yield

$$
N=\sigma(x) \sigma(y) \sigma(v-\mu) \sigma(x+y+\mu+v),
$$

and this formula, together with (A.9) and (A.7), yield

$$
\alpha(x+y)=\alpha(x) \alpha(y)+\frac{\alpha(x) \sigma(x)}{\sigma(x+v)} \frac{\alpha(y) \sigma(y)}{\sigma(y+v)} \frac{\sigma(v-\mu) \sigma(x+y+\mu+v)}{\sigma(\mu) \sigma(x+y+\mu)},
$$

which has just the functional form (3.15) and yields, via (3.13), precisely (3.21) and (3.22). Q.E.D.

Acknowledgements. It is a pleasure to thank O. Ragnisco for useful discussions. The research reported in this paper is supported in part by funds provided by the Italian Ministry of Education. 


\section{References}

1. Ruijsenaars, S.N.M., Schneider, H.: A new class of integrable systems and its relation to solitons, preprint, Mathematisches Institut, Universität Tübingen (to be published)

2. Lax, P.D.: Integrals of nonlinear equations of evolution and solitary waves. Commun. Pure Appl. Math. 21, 467-490 (1968)

3. Handbook of mathematical functions. Abramowitz, M., Stegun, I.A. (eds.). National Bureau of Standards. Applied mathematics series, Vol. 55, 1964. (Chap. 18: Weierstrass elliptic and related functions)

4. Bruschi, M., Ragnisco, O.: Private communication

5. Calogero, F.: Motion of poles and zeros of special solutions of nonlinear and linear partial differential equations and related "solvable" many body problems. Nuovo Cim. 43B, 177-241 (1978)

6. Calogero, F.: Integrable dynamical systems and related mathematical results. In: Nonlinear phenomena [Proc. of the CIFMO School and Workshop held at Oaxtepec, Mexico, November 29-December 17, 1982; Wolf, K.B. (ed.)]. Lecture Notes in Physics, Vol. 189. Berlin, Heidelberg, New York: Springer 1983, pp. 47-109

7. Calogero, F.: A class of solvable dynamical systems. Physica 18D, 280-302 (1986)

8. Calogero, F.: A class of integrable dynamical systems. Inverse Problems 1, L21-L24 (1985)

9. Calogero, F.: Integrable dynamical systems and some other mathematical results (remarkable matrices, identities, basic hypergeometric functions). In: Systèmes dynamiques non linéaires: integrabilité et comportement qualitatif. Winternitz, P. (ed.). SMS 102, Les Presses de l'Université de Montréal, 1986, pp. 40-70

Communicated by J. Mather

Received June 15, 1986 